\title{
RESULTATS PRELIMINAIRES DE L'OBSERVATION DES RADIOSOURCES A L'AIDE DE L'INTERFEROMETRE DE NANCAY
}

\author{
A. BOISCHOT \\ Observatoire de Meudon, Meudon (Seine-et-Oise), France
}

Un des problèmes majeurs de l'étude des radiosources est leur identification avec des objets optiques. Cette identification est presque toujours rendue très difficile par l'imprécision des mesures de position des radiosources.

Bien qu'il ait été spécialement conçu pour l'étude du soleil, le grand interféromètre de Nançay [1] a pu être utilisé pour préciser les coordonnées de quelques radiosources, en particulier les ascensions droites.

Nous donnons ici les résultats préliminaires de ces observations, qui seront complétées prochainement par une mesure des déclinaisons à l'aide de la branche nord-sud de l'interféromètre.

\section{CARACTERISTIQUES DE L'INTERFEROMETRE}

Interféromètre type Christiansen, 32 antennes.

Fréquence utilisée $169 \mathrm{Mc} / \mathrm{s}$.

Largeur du lobe à demi-puissance 3'.8.

Distance entre lobes principaux 2 degrés.

Surface totale des antennes $650 \mathrm{~m}^{2}$.

\section{METHODES DE MESURES}

(a) Ascension droite.-Elle est déterminée par la mesure du temps sidéral du passage de la source dans le lobe central de l'interféromètre. La position exacte de ce lobe a été fixée en observant le passage des radiosources Cassiopée A, Cygne A, Vierge A, Taureau A, dont les positions sont connues avec précision par des mesures optiques.

(b) Déclinaison.-On la déduit du temps de passage entre deux lobes principaux. La précision est très mauvaise pour les faibles déclinaisons, aussi donnons-nous dans le Tableau I les mesures de J. R. Shakeshaft et al. [2] pour les sources de déclinaison comprise entre -5 et +5 degrés.

(c) Diamètre. - Il est déterminé à partir de l'élargissement du lobe enregistré par rapport au lobe théorique. On l'exprime en "diamètre équivalent," diamètre d'un disque circulaire de luminance uniforme qui conduirait au même élargissement. Les sources de diamètre inférieur à une minute d'arc ne donneront pas d'élargissement sensible: nous ne pourrons donc pas en déterminer la valeur exacte.

(d) Densité de fux. - On mesure la surface enregistree lors du passage de la source dans le lobe de l'interféromètre. L'étalonnage a été fait à partir 
NO. 89

TABLEAU I

\begin{tabular}{|c|c|c|c|c|c|c|c|c|c|c|}
\hline $\mathbf{N}^{\circ}$ & & $\alpha_{1950}$ & & $\Delta \alpha$ & $\delta_{1950}$ & & $\Delta \delta$ & $\theta$ & $S$ & \\
\hline 1 & $\begin{array}{l}01^{\mathrm{h}} \\
01\end{array}$ & $\begin{array}{l}06^{\mathrm{m}} \\
06^{\mathrm{m}}\end{array}$ & $\begin{array}{l}04.5 \\
20\end{array}$ & $\begin{array}{l}1 \mathrm{~s} \\
5\end{array}$ & $\begin{array}{l}+13^{\circ} \\
+13\end{array}$ & $\begin{array}{l}01^{\prime} \\
03\end{array}$ & $\begin{array}{l}20^{\prime} \\
10\end{array}$ & $<1^{\prime}$ & 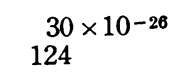 & $2 \mathrm{C} 94$ \\
\hline 2 & $\begin{array}{l}04 \\
04\end{array}$ & $\begin{array}{l}14 \\
15\end{array}$ & $\begin{array}{l}45.5 \\
12\end{array}$ & $\begin{array}{l}4 \\
5\end{array}$ & $\begin{array}{l}+38 \\
+38\end{array}$ & $\begin{array}{l}00 \\
01\end{array}$ & $\begin{array}{l}30 \\
10\end{array}$ & $\overline{<5}$ & $\overline{141}$ & $2 \mathrm{C} 379$ \\
\hline 3 & $\begin{array}{l}04 \\
04\end{array}$ & $\begin{array}{l}33 \\
33\end{array}$ & $\begin{array}{l}50.5 \\
57.5\end{array}$ & $\begin{array}{l}2 \\
1\end{array}$ & $\begin{array}{l}+29 \\
+29\end{array}$ & $\begin{array}{l}15 \\
32.5\end{array}$ & $\begin{array}{r}10 \\
3\end{array}$ & $<1$ & $\begin{array}{l}125 \\
400\end{array}$ & $\begin{array}{l}\text { 04N3A } \\
2 \mathrm{C} 404\end{array}$ \\
\hline 4 & $\begin{array}{l}05 \\
05\end{array}$ & $\begin{array}{l}01 \\
01\end{array}$ & $\begin{array}{l}11.5 \\
16.6\end{array}$ & 2.5 & $\begin{array}{l}+37 \\
+37\end{array}$ & $\begin{array}{l}50 \\
58\end{array}$ & $\begin{array}{r}15 \\
5\end{array}$ & $<1$ & $\begin{array}{r}60 \\
175\end{array}$ & $2 \mathrm{C} 440$ \\
\hline 5 & $\begin{array}{r}* 05 \\
05\end{array}$ & $\begin{array}{l}31 \\
31\end{array}$ & $\begin{array}{l}31.5 \\
30.5\end{array}$ & 1.5 & $\begin{array}{r}*+21 \\
+21\end{array}$ & $\begin{array}{l}59 \\
59.2\end{array}$ & 2 & $\begin{array}{l}4.6 \\
5\end{array}$ & $\begin{array}{r}700 \\
1850\end{array}$ & $\begin{array}{l}\text { 05N2A } \\
2 \mathrm{C} 481\end{array}$ \\
\hline 6 & $\begin{array}{l}06 \\
06\end{array}$ & $\begin{array}{l}14 \\
13\end{array}$ & $\begin{array}{l}40 \\
37\end{array}$ & $\begin{array}{r}10 \\
4\end{array}$ & $\begin{array}{r}\dagger+22 \\
+22\end{array}$ & $\begin{array}{l}38 \\
38\end{array}$ & $\begin{array}{l}5 \\
5\end{array}$ & $\overline{24}$ & $4 \overline{70}$ & $\begin{array}{l}\text { 06N2A } \\
2 \mathrm{C} 537\end{array}$ \\
\hline 7 & $\begin{array}{l}06 \\
06\end{array}$ & $\begin{array}{l}24 \\
24\end{array}$ & $\begin{array}{l}49 \\
42\end{array}$ & $\begin{array}{l}3 \\
7\end{array}$ & $\begin{array}{l}-05 \\
-05\end{array}$ & $\begin{array}{l}47 \\
47\end{array}$ & 15 & $\begin{array}{l}<1 \\
<7.5\end{array}$ & $\begin{array}{l}40 \\
93\end{array}$ & $2 \mathrm{C} 553$ \\
\hline 8 & $\begin{array}{l}08 \\
08\end{array}$ & $\begin{array}{l}09 \\
10\end{array}$ & $\begin{array}{l}54.5 \\
12\end{array}$ & $\begin{array}{l}3 \\
8\end{array}$ & $\begin{array}{l}+48 \\
+48\end{array}$ & $\begin{array}{l}20 \\
35\end{array}$ & $\begin{array}{r}10 \\
5\end{array}$ & $<1$ & $\begin{array}{r}30 \\
124\end{array}$ & $\begin{array}{l}\text { 08N4A } \\
\text { 2C724 }\end{array}$ \\
\hline 9 & $\begin{array}{l}09 \\
09\end{array}$ & $\begin{array}{l}15 \\
15\end{array}$ & $\begin{array}{l}46 \\
47\end{array}$ & $\begin{array}{l}1 \\
3\end{array}$ & $\begin{array}{l}-12 \\
-12\end{array}$ & $\begin{array}{l}00 \\
02\end{array}$ & $\begin{array}{r}30 \\
6\end{array}$ & $<1$ & $\begin{array}{l}200 \\
570\end{array}$ & $\begin{array}{l}\text { 09S1A } \\
2 \mathrm{C} 806\end{array}$ \\
\hline 10 & $\begin{array}{r}* 12 \\
12\end{array}$ & $\begin{array}{l}28 \\
28\end{array}$ & $\begin{array}{l}18 \\
18\end{array}$ & 3 & $\begin{array}{r}*+12 \\
+12\end{array}$ & $\begin{array}{l}40 \\
40\end{array}$ & 5 & $\begin{array}{l}6.1 \\
9\end{array}$ & $\begin{array}{r}740 \\
1700\end{array}$ & $\begin{array}{l}\text { 12N1A } \\
\text { 2C1041 }\end{array}$ \\
\hline 11 & $\begin{array}{l}14 \\
14\end{array}$ & $\begin{array}{l}09 \\
09\end{array}$ & $\begin{array}{l}31.5 \\
49\end{array}$ & $\begin{array}{l}4 \\
7\end{array}$ & $\begin{array}{l}+50 \\
+52\end{array}$ & $\begin{array}{l}23 \\
37\end{array}$ & $\begin{array}{r}10 \\
7\end{array}$ & $<2$ & $\begin{array}{r}35 \\
130\end{array}$ & $\begin{array}{l}\text { 14N5A } \\
\text { 2C1175 }\end{array}$ \\
\hline 12 & $\begin{array}{l}15 \\
15\end{array}$ & $\begin{array}{l}02 \\
02\end{array}$ & $\begin{array}{l}48.5 \\
48\end{array}$ & $\begin{array}{l}1.5 \\
6\end{array}$ & $\begin{array}{l}+26 \\
+26\end{array}$ & $\begin{array}{l}00 \\
02\end{array}$ & $\begin{array}{r}15 \\
4\end{array}$ & $\begin{array}{r}3 \\
<6\end{array}$ & $\begin{array}{r}60 \\
145\end{array}$ & $2 \mathrm{C} 1259$ \\
\hline 13 & $\begin{array}{l}15 \\
15\end{array}$ & $\begin{array}{l}08 \\
09\end{array}$ & $\begin{array}{l}34.5 \\
57\end{array}$ & $\begin{array}{l}4 \\
1.5\end{array}$ & $\begin{array}{l}+06 \\
+07\end{array}$ & $\begin{array}{l}30 \\
30\end{array}$ & 120 & $\stackrel{5 \pm 1}{-}$ & $\begin{array}{l}30 \\
78\end{array}$ & $2 \mathrm{C} 1270 ?$ \\
\hline 14 & $\begin{array}{l}15 \\
15\end{array}$ & $\begin{array}{l}14 \\
15\end{array}$ & $\begin{array}{l}10.5 \\
52\end{array}$ & $\begin{array}{r}3 \\
15\end{array}$ & $\begin{array}{l}+07 \\
+07\end{array}$ & $\begin{array}{l}00 \\
14\end{array}$ & $\begin{array}{l}60 \\
30\end{array}$ & $<1$ & $\begin{array}{l}40 \\
74\end{array}$ & $2 \mathrm{C} 1281 ?$ \\
\hline 15 & $\begin{array}{r}15 \\
\text { ou16 }\end{array}$ & $\begin{array}{l}54 \\
02\end{array}$ & $\begin{array}{l}07 \\
17.5\end{array}$ & 2 & +05 & 00 & 60 & $<1$ & 20 & \\
\hline 16 & 16 & 00 & 03 & 3 & +00 & & 180 & $<1$ & 20 & \\
\hline 17 & $\begin{array}{l}16 \\
16\end{array}$ & $\begin{array}{l}26 \\
27\end{array}$ & $\begin{array}{l}50.5 \\
00\end{array}$ & $\begin{array}{l}2.5 \\
5\end{array}$ & $\begin{array}{l}+39 \\
+39\end{array}$ & $\begin{array}{l}50 \\
42\end{array}$ & $\begin{array}{r}15 \\
6\end{array}$ & $<1$ & $\begin{array}{r}30 \\
155\end{array}$ & $\begin{array}{l}\text { 16N4A? } \\
\text { 2C1402 }\end{array}$ \\
\hline 18 & $\begin{array}{l}16 \\
16\end{array}$ & $\begin{array}{l}48 \\
48\end{array}$ & $\begin{array}{l}42 \\
41\end{array}$ & $\frac{1.5}{2}$ & $\begin{array}{l}+04 \\
+05\end{array}$ & $\begin{array}{l}00 \\
06\end{array}$ & $\begin{array}{r}60 \\
8\end{array}$ & $<^{2.5}$ & $\begin{array}{l}200 \\
900\end{array}$ & $\begin{array}{l}\text { 16N0A } \\
2 \mathrm{C} 1432\end{array}$ \\
\hline 19 & $\begin{array}{l}17 \\
17\end{array}$ & $\begin{array}{l}18 \\
18\end{array}$ & $\begin{array}{l}01 \\
00\end{array}$ & 1.5 & $\begin{array}{r}00 \\
+00\end{array}$ & 53 & $\begin{array}{r}120 \\
4\end{array}$ & $\begin{array}{c}5 \pm 1 \\
6\end{array}$ & $\begin{array}{l}140 \\
585\end{array}$ & $2 \mathrm{C} 1473$ \\
\hline 20 & $\begin{array}{l}18 \\
18\end{array}$ & $\begin{array}{l}28 \\
28\end{array}$ & $\begin{array}{l}19 \\
15\end{array}$ & $\begin{array}{l}4 \\
7\end{array}$ & $\begin{array}{l}+48 \\
+48\end{array}$ & $\begin{array}{l}45 \\
36\end{array}$ & $\begin{array}{l}5 \\
6\end{array}$ & $2 \pm 1$ & $\begin{array}{r}70 \\
138\end{array}$ & $2 C 1569$ \\
\hline 21 & $\begin{array}{r}* 19 \\
19\end{array}$ & $\begin{array}{l}57 \\
57\end{array}$ & $\begin{array}{l}44.5 \\
45.3\end{array}$ & 1 & $\begin{array}{r}*+40 \\
+40\end{array}$ & $\begin{array}{l}36 \\
35\end{array}$ & 1 & $\begin{array}{l}2.7 \\
2.3\end{array}$ & $\begin{array}{r}5000 \\
14000\end{array}$ & $\begin{array}{l}\text { 19N4A } \\
\text { 2C1673 }\end{array}$ \\
\hline 22 & $\begin{array}{l}20 \\
20\end{array}$ & $\begin{array}{l}12 \\
13\end{array}$ & $\begin{array}{l}15 \\
50\end{array}$ & $\begin{array}{r}1 \\
10\end{array}$ & $\begin{array}{l}+23 \\
+23\end{array}$ & $\begin{array}{l}25 \\
36\end{array}$ & $\begin{array}{l}15 \\
20\end{array}$ & $<1$ & $\begin{array}{r}40 \\
192\end{array}$ & $2 \mathrm{C} 1686 ?$ \\
\hline 23 & $\begin{array}{l}22 \\
22\end{array}$ & $\begin{array}{l}11 \\
12\end{array}$ & $\begin{array}{l}48.5 \\
01\end{array}$ & $\begin{array}{l}2 \\
5\end{array}$ & $\begin{array}{l}-16 \\
-17\end{array}$ & $\begin{array}{l}10 \\
16\end{array}$ & $\begin{array}{r}20 \\
8\end{array}$ & $\stackrel{3 \pm 1}{<7.5}$ & $\begin{array}{r}40 \\
202\end{array}$ & $2 \mathrm{C} 1843$ \\
\hline 24 & $\begin{array}{l}23 \\
23\end{array}$ & $\begin{array}{l}14 \\
14\end{array}$ & $\begin{array}{l}05 \\
03\end{array}$ & $\frac{1.5}{5}$ & $\begin{array}{r}t+03 \\
+03\end{array}$ & $\begin{array}{l}51 \\
51\end{array}$ & 6 & $\begin{array}{l}<1 \\
<8\end{array}$ & $\begin{array}{l}25 \\
62\end{array}$ & $2 \mathrm{C} 1883$ \\
\hline 25 & $\begin{array}{r}* 23 \\
23\end{array}$ & $\begin{array}{l}21 \\
21\end{array}$ & $\begin{array}{l}11.5 \\
12\end{array}$ & 1 & $\begin{array}{r}*+58 \\
+58\end{array}$ & $\begin{array}{l}32 \\
32\end{array}$ & 0.7 & $\begin{array}{l}5.5 \\
3.8\end{array}$ & $\begin{array}{r}8000 \\
23200\end{array}$ & $\begin{array}{l}\text { 23N5A } \\
\text { 2C1890 }\end{array}$ \\
\hline
\end{tabular}

* Positions déterminées optiquement.

† Valeurs déterminées par Shakeshaft et al. [2]. 
des densités de flux des sources Cassiopée $A$, Cygne A, et Vierge A donnée par Seeger, la valeur absolue du flux sur $169 \mathrm{Mc} / \mathrm{s}$ pour Cassiopée A $(80 \times$ $10^{-24}$ watts $\left.\mathrm{m}^{-2}(\mathrm{c} / \mathrm{s})^{-1}\right)$ étant obtenue en intrapolant les valeurs obtenues par Hagen $(193 \mathrm{Mc} / \mathrm{s})$ et Brown et Hazard (158 Mc/s) [3].

La précision sur les valeurs relatives est de l'ordre de 5 pour cent pour les sources intenses, moindre pour les sources plus faibles.

Une description détaillée de l'interféromètre et une discussion des méthodes employées ont été publiées par ailleurs [4].

(e) Remarques.-Le pouvoir séparateur étant de 3'.8, l'interféromètre sera très peu sensible aux sources faibles et larges. Il sera complètement insensible aux sources de diamètre supérieur a 2 degrés.

Nous avons cherché a obtenir avec le maximum de précision la position d'un petit nombre de radiosources. Ceci nous a conduit à rejeter quelques sources assez intenses pour lesquelles la confusion avec une source voisine empêchait une mesure précise. En particulier, nous n'avons pas fait figurer dans notre liste les sources voisines du centre galactique. L'interféromètre nous permet d'en distinguer au moins six, dont plusieurs de diamètre important, mais l'effet de confusion nous empéche d'en déterminer les positions avec précision. Nous pouvons seulement dire actuellement que nous observons une émission dans la direction du centre galactique, correspondant à une source large (probablement de l'ordre de 30 minutes d'arc) dont l'ascension droite est $17^{\mathrm{b}} 42^{\mathrm{m}} 45^{\mathrm{a}} \pm 10^{\mathrm{s}}$.

D'autre part, ces observations préliminaires sont limitées aux déclinaisons comprises entre -20 et +60 degrés.

Les positions des sources du Taureau, de l'Hydre, de la Vierge, du Cygne, et de Cassiopée ayant été utilisées pour la détermination de la position du lobe de l'interférometre, nous donnons dans notre liste les positions observées optiquement.

\section{RESULTATS}

Le Tableau I indique pour 25 sources: l'ascension droite $\alpha$; la déclinaison $\delta$, le diametre apparent $\theta$, et le flux $\boldsymbol{\theta}$, et les erreurs sur les positions. Sous chacune des valeurs observees, nous avons indiqué les valeurs correspondantes trouvees par Shakeshaft [2].

Les positions obtenues pour 25 sources sont en général en bon accord avec celles du Catalogue de Cambridge sur $81.5 \mathrm{Mc} / \mathrm{s}$ [2]. Les sources indiquées par ce catalogue ayant un diamètre supérieur a 20 minutes d'arc ne sont pas détectées. Au contraire la presque totalité des sources étroites de densité de flux supérieure à $80 \times 10^{-20}$ watts $\mathrm{m}^{-2}(\mathrm{c} / \mathrm{s})^{-1}$ sur $81.5 \mathrm{Mc} / \mathrm{s}$ sont observées sur $169 \mathrm{Mc} / \mathrm{s}$. Elles ont presque toutes un diametre inférieur à une minute d'arc.

Deux sources assez intenses (2C 855 et 2C 1012) n'ont pas été observées. Si la position déterminée sur $81.5 \mathrm{Mc} / \mathrm{s}$ est exacte, leur amplitude est probablement surestimée ou leur spectre très anormal.

D'autre part nos sources $13,14,15$, et 16 ne figurent pas dans le catalogue de Cambridge, mais celui-ci signale plusieurs sources dans la même région (2C 1270, 1281, 1289, 1291). La proximité de ces sources a pu gêner la determination précise des positions avec un instrument à pouvoir séparateur insuffisant. 
Signalons enfin qu'il est difficile de comparer nos résultats à ceux de Mills et Slee. Dans la liste préliminaire qui a été publiée [5], il n'y a qu'une source étroite suffisamment intense pour pouvoir être détectée avec notre interféromètre. Il est à noter alors que les positions données par les trois catalogues sont en bon accord (Tableau II).

\begin{tabular}{|c|c|c|c|c|c|c|c|c|}
\hline \multicolumn{9}{|c|}{ TABLEAU II } \\
\hline & & $\alpha_{1950}$ & $\Delta \alpha$ & $\delta_{19}$ & & $\Delta \delta$ & $S$ & $d$ \\
\hline Ryle & $06^{\mathrm{h}}$ & $24^{\mathrm{m}} 42^{\mathrm{s}}$ & $7 \mathrm{~s}$ & $-05^{\circ}$ & $47^{\prime}$ & $15^{\prime}$ & $93 \times 10^{-20}$ & 7.5 \\
\hline Mills & 06 & $24 \quad 48$ & 6 & -05 & 57 & 03 & 130 & - \\
\hline Boischot & 06 & $24 \quad 49$ & 3 & - & & - & 40 & $<1$ \\
\hline
\end{tabular}

\section{REFERENCES}

[1] Blum, E. J., Boischot, A., et Ginat, M. Ann. Astrophys. 20, 155, 1957.

[2] Shakeshaft, J. R. et al. Mem. R.A.S. 67, 106, 1955.

[3] Hagen, J. P. Radio Astronomy (I.A.U. Symposium No. 4, 1955). Cambridge, England, 1957, p. 142.

[4] Boischot, A. Thèse, Paris 1958. (A paraître dans Ann. Astrophys.)

[5] Mills, B. Y., et Slee, O. B. Aust. J. Phys. 10, 162, 1957.

\section{Discussion}

Denisse: I should like to mention the way in which Blum intends to expand his 32-antenna interferometer, by putting at each end an aerial of aperture equal to the distance between individual mirrors. The output of this twoaerial interferometer will be connected with the output of the 32 aerials. This device has a square spatial bandwidth, with no gaps except for the zero frequency. Covington uses a similar device.

Shakeshaft: The comparison of positions of intense sources from the $3 \mathrm{C}$ survey and Mills and Slee's survey shows a scatter of about $10^{\mathrm{s}}$ in right ascension and about 10 minutes of arc in declination. 